Research Article

\title{
Remedying the Mitochondria to Cure Human Diseases by Natural Products
}

\author{
Jian-Kang Mu, ${ }^{1}$ Yan-Qin Li, ${ }^{1}$ Ting-Ting Shi, ${ }^{2}$ Li-Ping Yu, ${ }^{1}$ Ya-Qin Yang, ${ }^{1}$ Wen Gu, \\ Jing-Ping $L i,{ }^{1}$ Jie $Y u \mathbb{D}^{1}$, and Xing-Xin Yang $\mathbb{C}^{1}$ \\ ${ }^{1}$ College of Pharmaceutical Science, Yunnan University of Chinese Medicine, 1076 Yuhua Road, Kunming 650500, China \\ ${ }^{2}$ Department of Pharmaceutical Preparation, The Xixi Hospital of Hangzhou Affiliated to Zhejiang University of Traditional \\ Chinese medicine, Hangzhou 310023, China
}

Correspondence should be addressed to Jie Yu; cz.yujie@gmail.com and Xing-Xin Yang; yxx78945@163.com

Received 4 April 2020; Revised 5 June 2020; Accepted 25 June 2020; Published 14 July 2020

Academic Editor: Ratanesh K. Seth

Copyright (c) 2020 Jian-Kang Mu et al. This is an open access article distributed under the Creative Commons Attribution License, which permits unrestricted use, distribution, and reproduction in any medium, provided the original work is properly cited.

\begin{abstract}
Mitochondria are the 'engine' of cells. Mitochondrial dysfunction is an important mechanism in many human diseases. Many natural products could remedy the mitochondria to alleviate mitochondria-involved diseases. In this review, we summarized the current knowledge of the relationship between the mitochondria and human diseases and the regulation of natural products to the mitochondria. We proposed that the development of mitochondrial regulators/nutrients from natural products to remedy mitochondrial dysfunction represents an attractive strategy for a mitochondria-involved disorder therapy. Moreover, investigating the mitochondrial regulation of natural products can potentiate the in-depth comprehension of the mechanism of action of natural products.
\end{abstract}

\section{Introduction}

As an important organelle in the cells, the mitochondria are considered the main powerhouse of the cells, because they can apply glucose, fatty acids, and certain amino acids as fuel sources to produce ATP through oxidative phosphorylation [1]. The mitochondria also play a critical role in many other processes, such as reactive oxygen species generation, maintenance of calcium homeostasis, adjustment of apoptotic cell death, regulation of lipid metabolism, and autophagy [2]. Thus, mitochondrial dysregulation of any form may lead to a variety of human diseases [2]. Mitochondrial dysfunction has been implicated in neurodegenerative disorders, cancer, liver diseases, myocardial injury, diabetes, and obesity $[3,4]$.

Natural products, including mixture and monomer, have been widely used to treat mitochondria-related diseases and have been reported as a highly significant source for the exploration of promising drugs/nutrients that have led to novel compounds for alleviating mitochondria-involved dis- orders, such as compounds with antitumor, neuroprotective, cardioprotective, hepaticprotective, antidiabetes, and antiobesity agents. The chemical synthesis of new drugs has rapidly developed in recent years with the advancement of combinatorial chemistry and computer-aided drug design technology [5]. However, due to the novel structures, therapeutic abilities, and certain unique pharmacological effects of the chemicals in natural products, the exploration of drugs and lead compounds from natural products is still an important approach for drug development [6].

The focus of this review was on mitochondrial regulation with natural products to treat human diseases. The purpose of this review was to examine the current knowledge of the relationship between mitochondria and human diseases and the regulation of natural products to the mitochondria. We proposed that the development of mitochondrial regulators/nutrients from natural products to remedy mitochondrial dysfunction represented attractive strategies for treating mitochondria-involved disorders. Moreover, investigating 
mitochondrial regulation of natural products can potentiate the in-depth comprehension of the underlying mechanism of action of natural products.

\section{Remedying the Mitochondria to Cure Human Diseases by Natural Products}

2.1. Regulating the Mitochondria to against Cancer. Prevention of cell death is a hallmark of human cancers and a major cause of treatment failure [7]. The mitochondria control the activation of apoptotic effects or mechanisms by regulating the translocation of proapoptotic proteins from the mitochondrial intermembrane space to the cytosol [8]. In addition, the mitochondria play an important role in various forms of nonapoptotic cell death and, especially, in necroptosis [7]. Because of their role in the regulation of basic cellular functions, it is not surprising that the mitochondria are involved in many aspects of tumorigenesis and tumor progression. For example, mutations in mitochondrial DNA that affect the compositions of the mitochondrial respiratory chain will lead to ROS overproduction, inefficient ATP production, and oxidative damage to the mitochondria and other macromolecules (including DNA), thus favoring chromosomal instability and carcinogenesis [9]. Furthermore, extensive polymorphisms and mutations in the mitochondrial DNA correlated with an increased risk of developing various malignancies [10]. Therefore, inducing cancer cells to undergo mitochondrial lesions and loss of function has become a very important direction in the field of anticancer drugs.

A large number of studies have shown that natural products have a significant anticancer activity by regulating the mitochondrial function with the following main mechanisms (Table 1): (1) promote the release of proapoptotic factors and induce tumor cell apoptosis by changes in mitochondrial membrane permeability, regulation of Bcl-2 family proteins, and other pathways; (2) regulate the mitochondrial energy metabolism, including the respiratory chain and tricarboxylic acid cycle; and (3) increase ROS levels and enhances oxidative damage.

2.2. Regulating the Mitochondria to against Neurodegenerative Diseases. Neurodegenerative diseases, such as Alzheimer's disease, Parkinson's disease, Huntington's disease, amyotrophic lateral sclerosis, and Friedreich's ataxia, are strongly age related and currently cannot be cured [11]. In neurons, efficient clearance of injured mitochondria through mitophagy plays a fundamental role in mitochondrial and metabolic homeostases and neuronal survival and health [11]. The mitochondria are organized in a highly dynamic tubular network that is continuously reshaped by opposing processes of fusion and fission [12]. Defects in fusion or fission will result in mitochondrial fragmentation, reduce energy metabolism, and increase oxidative stress, thus accelerating cell dysfunction and death, leading to neurodegenerative disease [13]. Therefore, the regulation of mitochondrial dynamics, such as fusion, fission, and mitochondrial phagocytosis, represents a significant avenue for controlling the fate of neurons $[12,13]$.

Through numerous animal experiments and clinical studies, a variety of drugs from natural products were identi- fied with neuroprotective effects. Many of these drugs can exert neuroprotective effects by protecting the mitochondrial function (Table 2): (1) regulate $\Delta \Psi \mathrm{m}$ and membrane fluidity; (2) protect mitochondrial structure and morphology; (3) regulate mitochondrial apoptotic pathways, reduce the release of proapoptotic factors, and inhibit neuronal apoptosis; (4) improve the cellular mitochondrial respiratory function (energy metabolism); (5) enhance superoxide dismutase (SOD) activity, inhibit oxidative stress, and reduce ROS damage; and (6) improve mitophagy.

2.3. Regulating the Mitochondria to Remedy Liver Diseases. The liver, an organ with high energy requirements, plays a pivotal role in the synthesis and secretion of multiple endogenous compounds. Liver functioning is highly dependent on the mitochondria producing ATP for biosynthetic and detoxifying properties [14]. In previous studies, it was suggested that mitochondrial dysfunction is a critical factor in the initiation and progression of liver diseases, including ischemia/reperfusion (IR) injury, nonalcoholic/alcoholic fatty liver disease (NAFLD/AFLD), nonalcoholic/alcoholic steatohepatitis (NASH/ASH), and hepatic fibrosis, as well as intoxications by xenobiotics or heavy metals, bacterial, viral, and parasitic infections [15]. The mitochondria play an important role in the process of hepatic apoptosis and necrosis. The degree of the mitochondrial activity in the liver directly affects liver function [16].

In previous studies, it was shown that some natural medicines can protect liver cells from damage or liver fibrosis by protecting the mitochondrial function (Table 3): (1) stabilize the fluidity of mitochondrial membranes and protect the structure and morphology of liver mitochondria; (2) regulate the mitochondrial apoptotic pathway, reduce the release of proapoptotic factors, and inhibit hepatocyte apoptosis; (3) increase the mitochondrial energy metabolism; and (4) enhance SOD activity, inhibit oxidative stress, and reduce ROS damage.

2.4. Regulating the Mitochondria to against Diabetes and Its Complications. Diabetes mellitus (DM) is one of the most common metabolic diseases worldwide [17]. Patients with DM display hyperglycemia induced by a damage in insulin secretion (type 1), insulin action (type 2), or both. Type 1 diabetes mellitus (T1DM), which accounts for less than $10 \%$ of diabetes cases, is characterized by an immune-mediated destruction of $\beta$ cells in the pancreatic islets of Langerhans, resulting in insulin deficiency [18]. Type 2 diabetes mellitus (T2DM), which accounts for less than $90 \%$ of diabetes cases, involves insulin resistance (IR) in peripheral tissues and increased levels of blood glucose, because of overnutrition with an insulin secretion defect $[18,19]$. IR continuously exists in the development of T2DM. A defect in the secretion function of pancreatic beta-cell is the prerequisite of T2DM development [20]. Mitochondrial dysfunction is the common mechanism of IR and injury of secretion function of pancreatic beta-cell $[20,21]$. Furthermore, many mitochondrial gene mutation sites related to diabetes have been found, and the $3243 \mathrm{~A} \rightarrow \mathrm{G}$ mutation in the mtDNA tRNA ${ }^{\text {Leu(UUR) }}$ gene is the most common cause of mitochondrial diabetes 


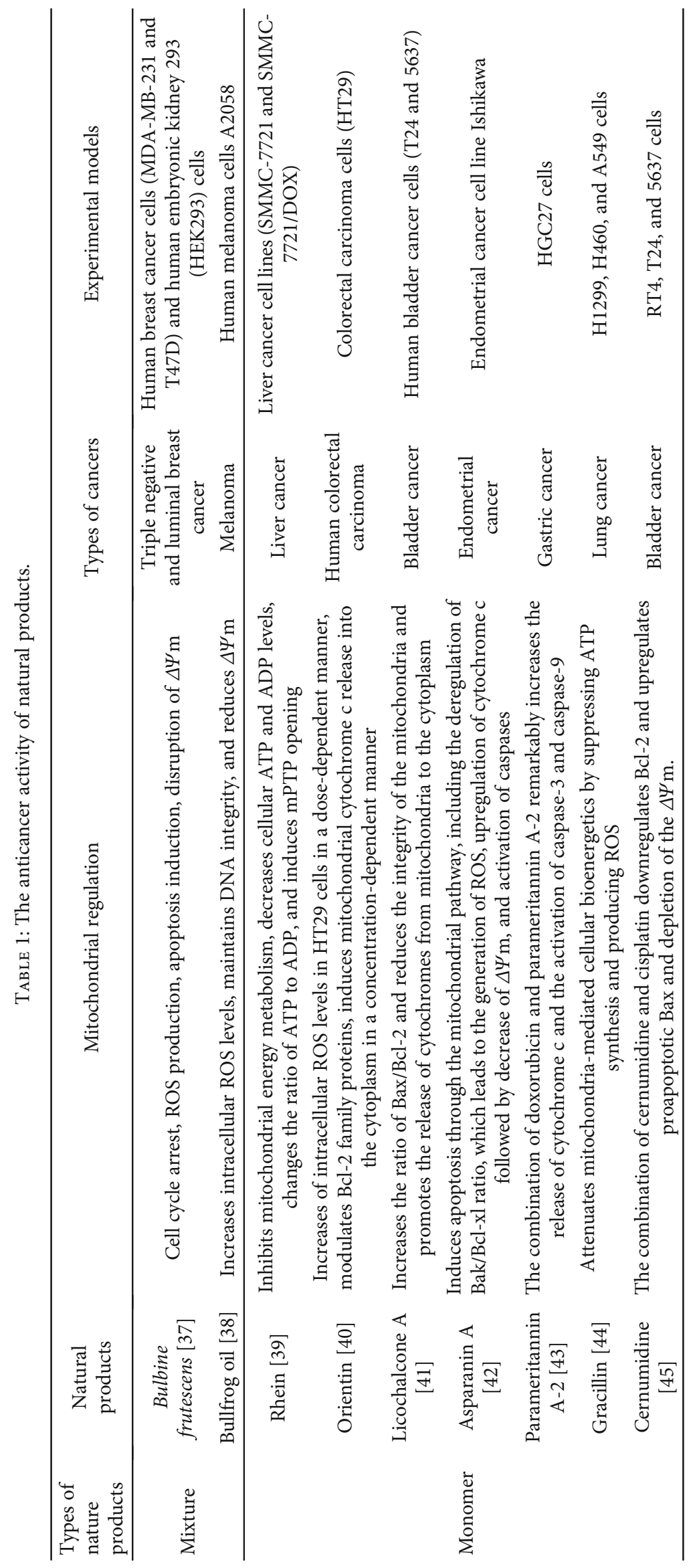




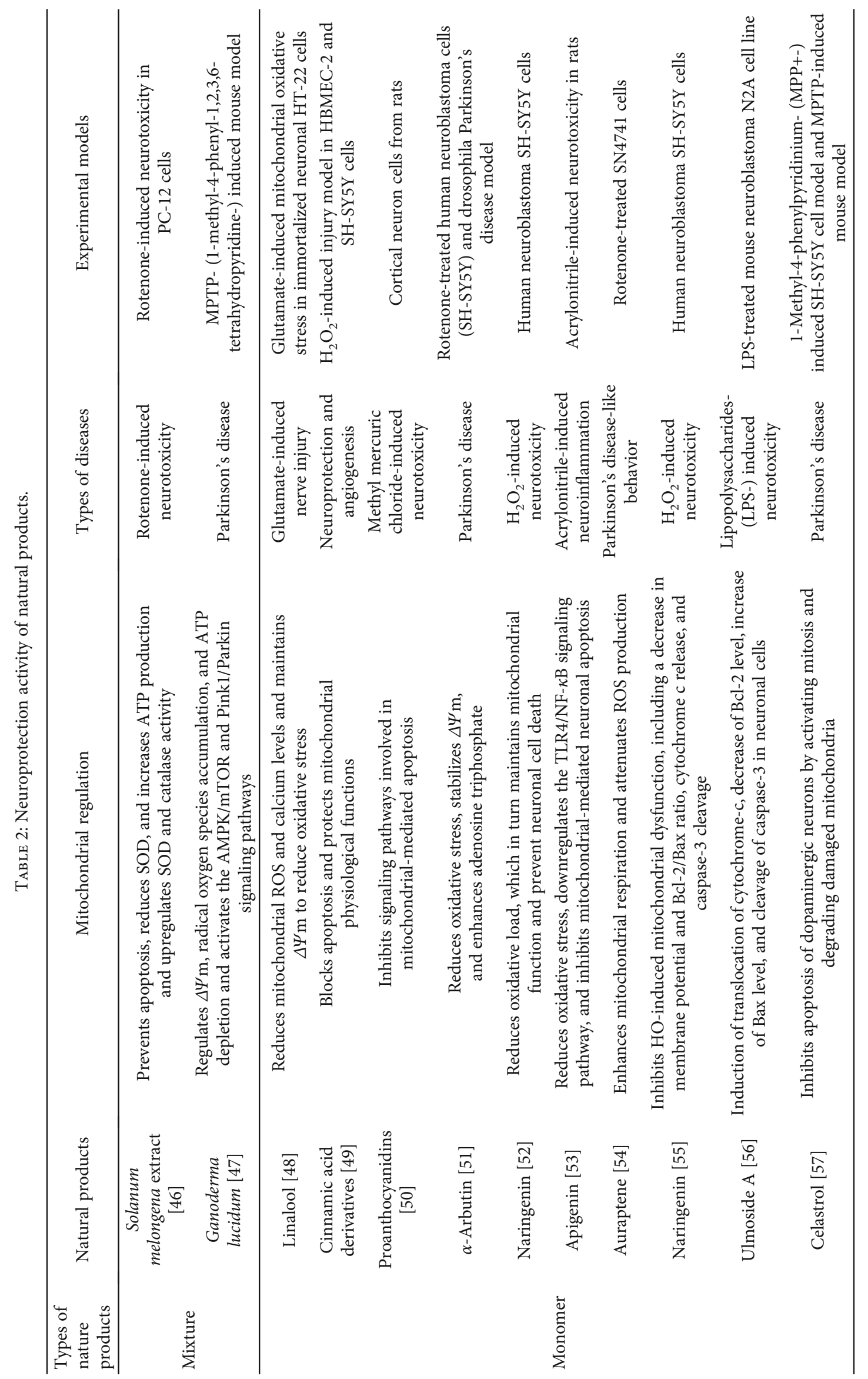




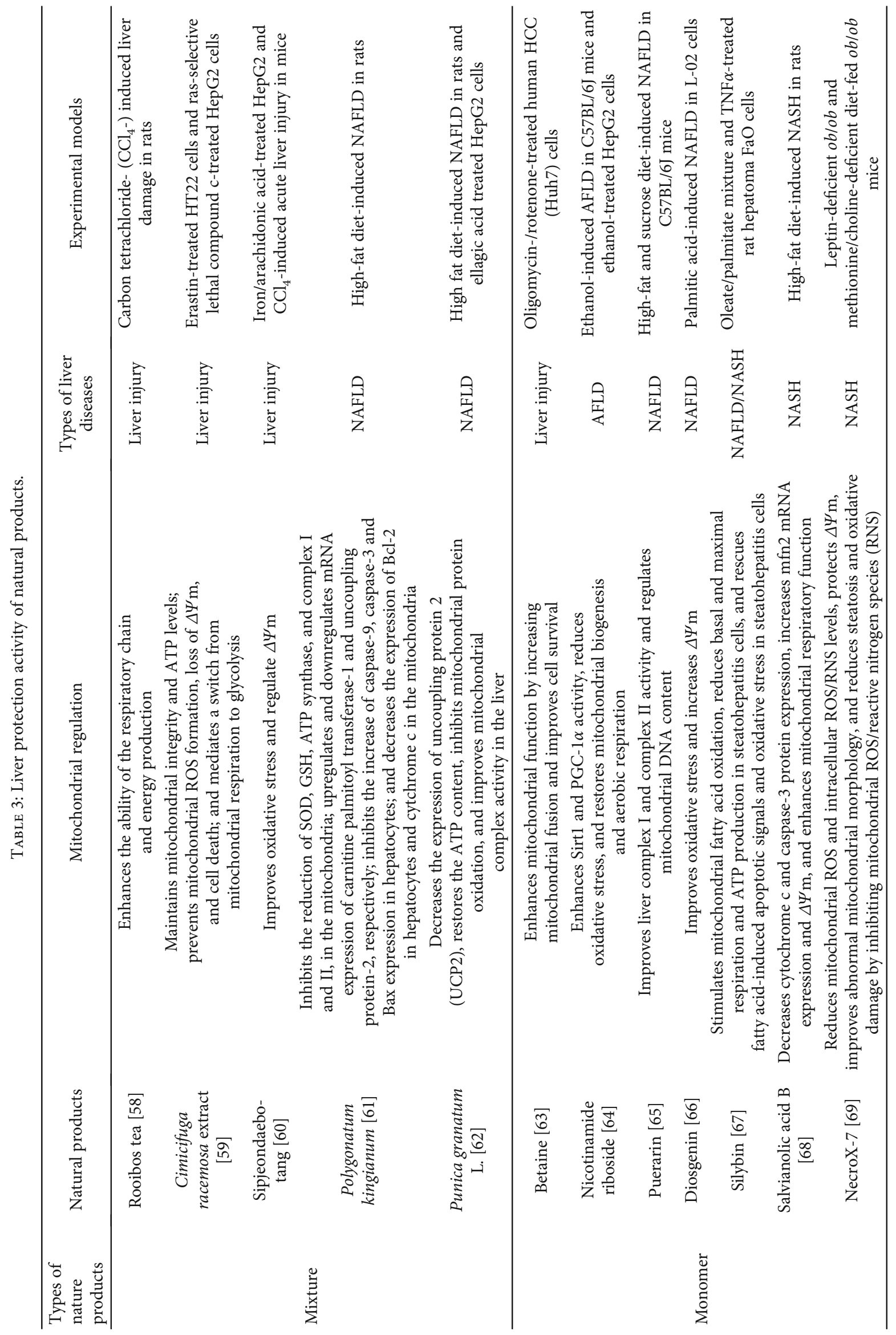


[22]. This mutation results in the reduction of insulin release and insulin resistance and leads to persistent hyperglycemia, which in turn causes mitochondrial dysfunction and reduces insulin release [22]. Muscle biopsies of diabetic patients have revealed abnormal mitochondrial metabolism and reduced mitochondria quantity $[23,24]$.

A large proportion of the diabetic population develops chronic vascular complications leading to significant morbidity and mortality [25]. Microvascular complications include diabetic nephropathy, neuropathy, and retinopathy; muscle atrophy, coronary, and peripheral vascular diseases; and stroke [25]. The hyperglycemic milieu alters the epigenetic machinery and mtDNA. Other genes associated with mitochondrial homeostasis are epigenetically modified, thereby further contributing to mitochondrial damage [26]. Dysfunction is seen in the context of an altered mitochondrial metabolism and oxygen consumption, increased oxidative stress, and alterations to mitochondrial networking and turnover. An increasing body of evidence has highlighted the role of mitochondrial dysfunction in the development of diabetic complications $[27,28]$.

In previous studies, it was found that many natural products alleviated the symptoms of T2DM and its complications by protecting the mitochondrial function (Tables 4 and 5): (1) protecting the structure and morphology of the mitochondria from pathological organs/tissues; (2) regulating the mitochondrial apoptotic pathway, reducing the release of proapoptotic factors, and inhibiting cell apoptosis; (3) increasing mitochondrial energy metabolism; and (4) enhancing SOD activity, inhibiting oxidative stress, and reducing ROS damage.

2.5. Regulating the Mitochondria to Antiobesity. Obesity is caused by an imbalance between energy intake and expenditure and results in excessive energy that in adipose tissue is stored as triglycerides (TGs) [29]. It is not only recognized as a simple condition but also causes many metabolic diseases, such as cardiovascular disease, T2DM, hypertension, and fatty liver disease [30]. In many organs and tissues (including adipose tissue), the mitochondria are center stage in the control of energy homeostasis. Research evidence indicates that mitochondrial dysfunction in adipocytes is closely related to obesity [31]. Various physiological conditions, such as excessive nutrition and genetic factors, disrupt mitochondrial function by impairing mitochondrial biogenesis, dynamics, and oxidative capacity. Mitochondrial dysfunction in adipocytes may have impact on adipogenesis and insulin sensitivity and may significantly alter their metabolic function, which ultimately leads to obesity [32].

Animal experiments and clinical studies have successively identified many drugs from natural products for treating obesity. Many of these drugs can regulate mitochondrial function to treat obesity, primarily through promoting energy and fat metabolism (Table 6).

2.6. Regulating the Mitochondria to against Myocardial Injury. Myocardial injury can be caused by myocardial infarction, ischemia, inflammatory cell infiltration, poisoning, and so on [33]. The essence of myocardial injury refers to the edema, degeneration, and necrosis of myocardial cells; the breakdown and lysis of myofibrils; and cellular structures, such as mitochondria in severe lesions. Severe myocardial injury can lead to myocarditis and heart failure [34]. Myocardium is the most energy consuming tissue in the human body [35]. Mitochondrial abnormalities play a central role in the pathogenesis and development of various heart diseases, including acute myocardial infarction and cardiomyopathy [36].

In previous studies, it was shown that natural products can protect the heart by regulating the mitochondrial function (Table 7): (1) stabilize $\Delta \Psi \mathrm{m}$ and membrane fluidity; (2) protect mitochondrial structure and morphology; (3) adjust mitochondrial apoptotic pathways, reduce the release of proapoptotic factors, and inhibit myocardial cell apoptosis; (4) improve mitochondrial energy metabolism; and (5) enhance SOD and GSH activity, inhibit oxidative stress, and reduce ROS damage.

\section{Similarities and Differences between the Mitochondrial Mechanisms for Natural Products Regulating Different Diseases}

As shown in Table 8, there are some common mechanisms in mitochondrial dysfunction among different diseases, and the similarities and differences existed between the mitochondrial mechanisms for natural products regulating different diseases. For instance, almost all the mitochondriainvolved diseases, including neurodegenerative disorders, cancer, liver diseases, myocardial injury, diabetes, and obesity, are related with mitochondrial energy metabolism, which can be remedied by natural products. However, fatty acid oxidation is specifically involved with obesity and fatty liver disease, which can also be regulated by natural products. Furthermore, a variety of natural products can remedy the mitochondria through multiple mechanisms to cure various diseases.

\section{Conclusion}

Mitochondria are cytoplasmic organelles responsible for cell survival and cell death. Mitochondrial dysfunction has been reported to be involved in many diseases. Many natural products can regulate the mitochondria in various ways to alleviate related diseases (Figure 1). However, only a few have become clinical drugs for treating patients, and many compounds have not been used in clinical practice. Additional studies (such as pharmacodynamics, toxicology, and structure-activity relationship) of these compounds should be performed, which will promote that more natural products will be available for clinical usage. In addition, the monomers that can regulate the mitochondria in many natural extracts remain unclear, and further studies are warranted to identify natural monomers that can regulate the mitochondria. With the deepening of research, it is believed that more natural products that can regulate the mitochondria have the potential to be used in treating diseases, which is of utmost importance. 


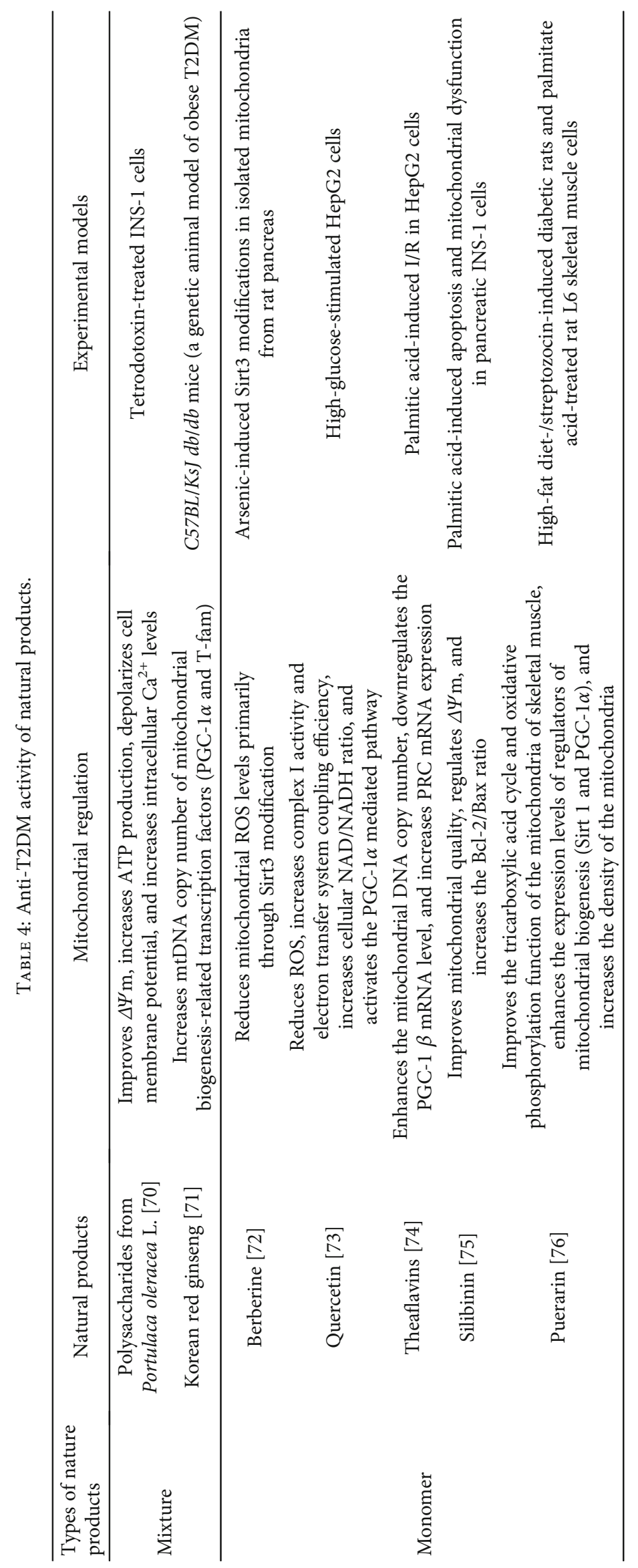




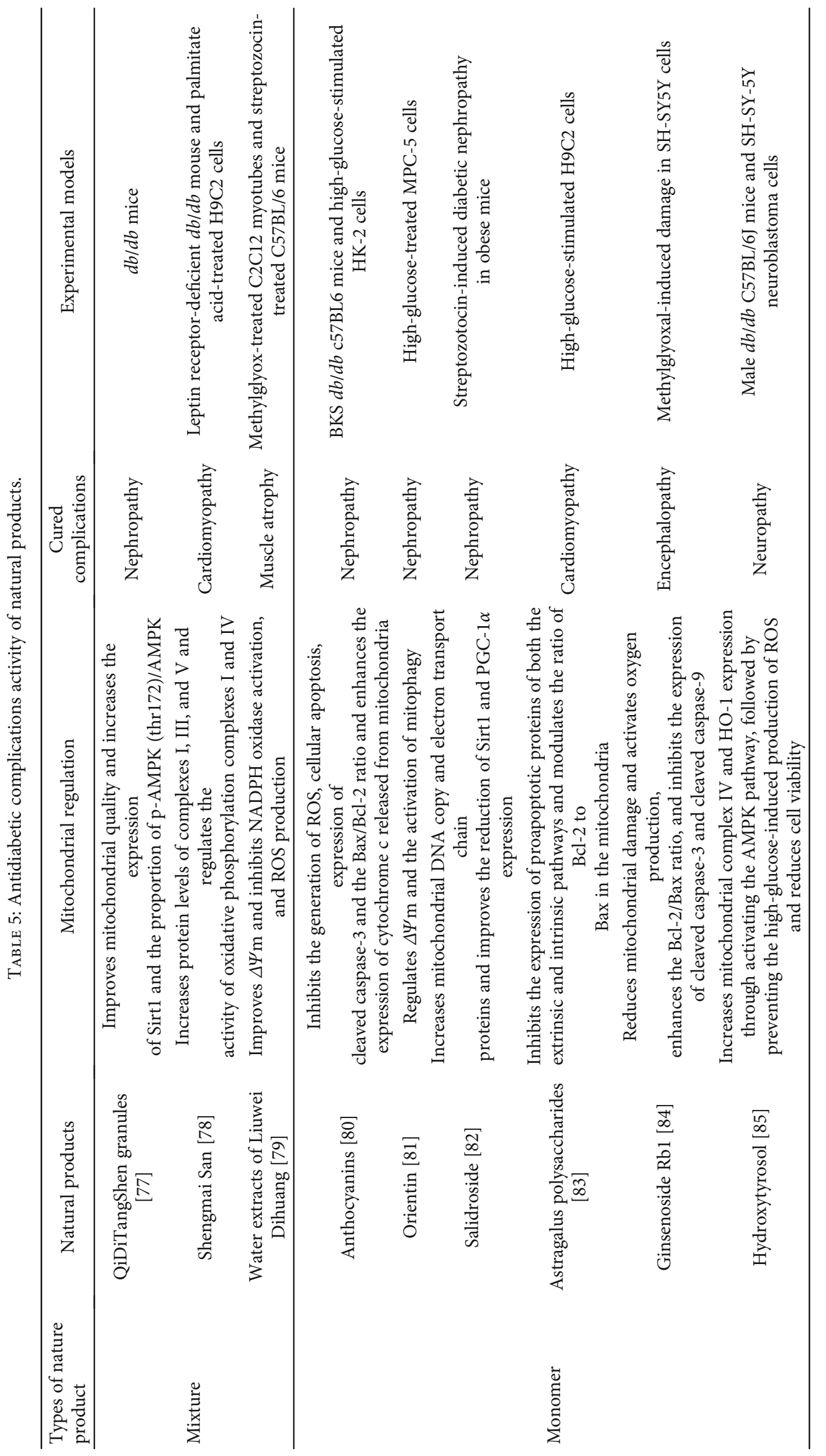




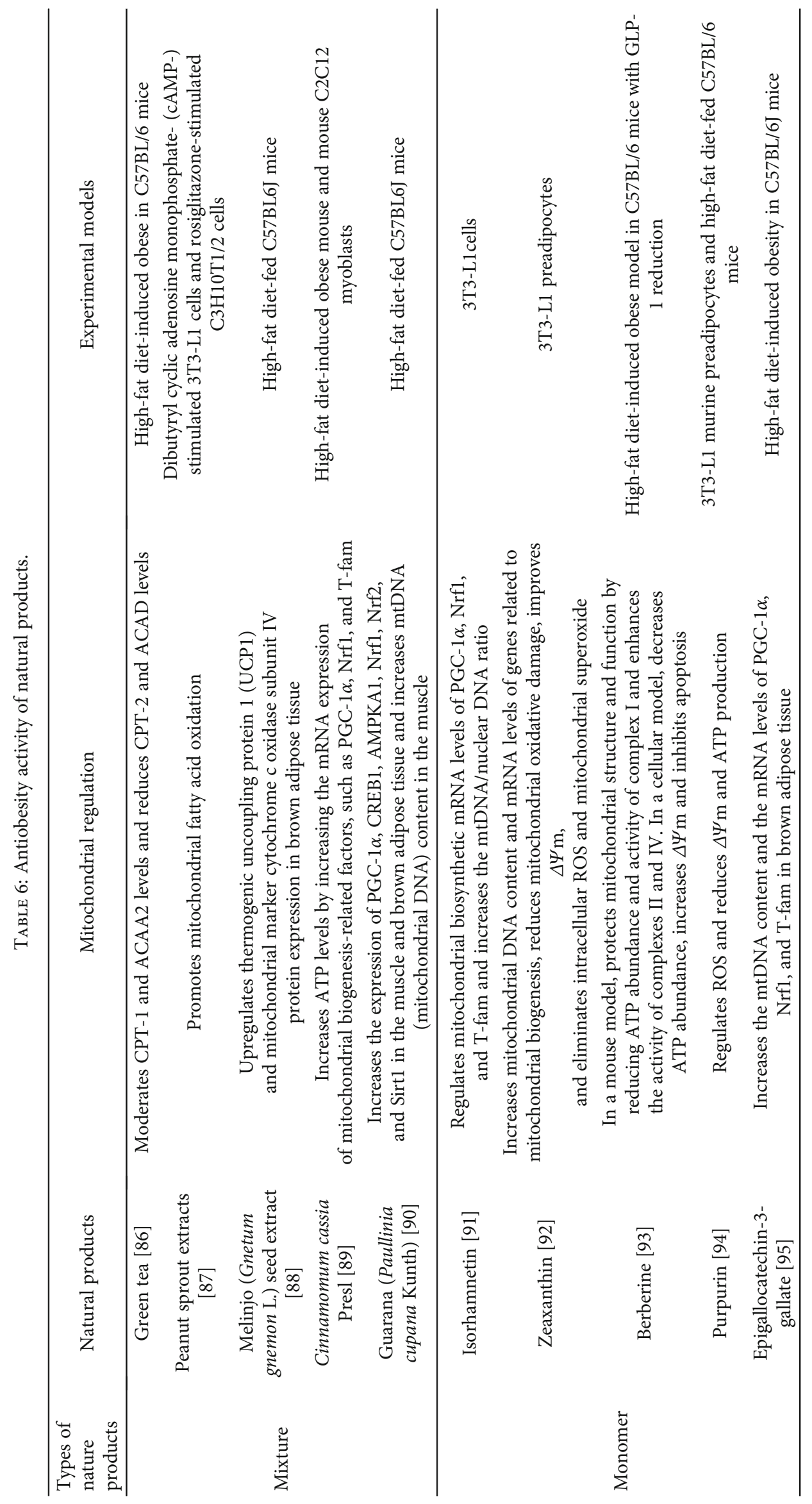




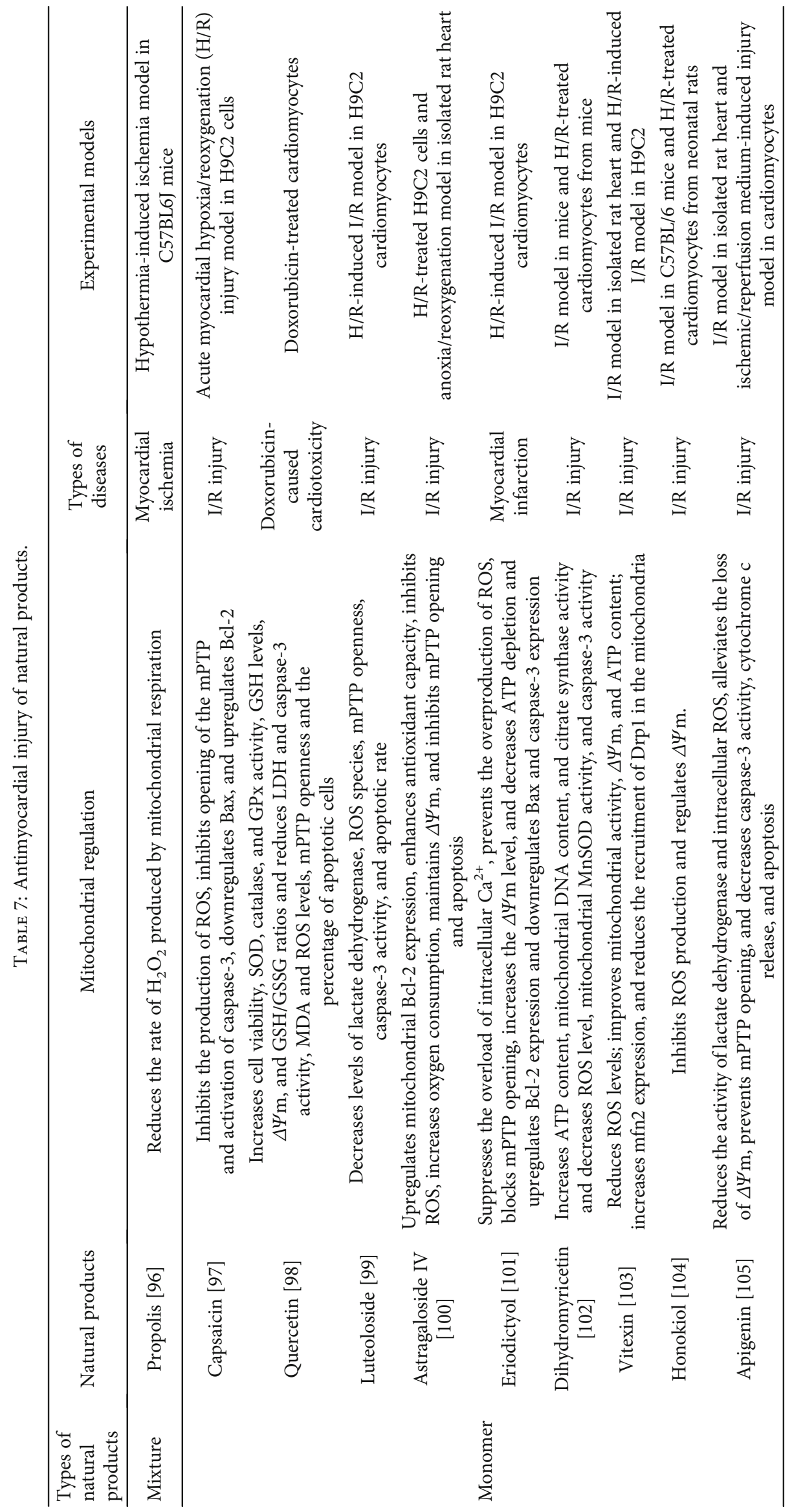


TABLE 8: Similarities and differences between the mitochondrial mechanisms for natural products regulating different diseases.

\begin{tabular}{|c|c|c|}
\hline Diseases & Major mechanisms & Natural products \\
\hline \multirow{17}{*}{ Cancer } & \multirow{2}{*}{ Energy metabolism obstruction } & Rhein [39] \\
\hline & & Gracillin [44] \\
\hline & \multirow{5}{*}{ Oxidative stress } & Bulbine frutescens [37] \\
\hline & & Bullfrog oil [38] \\
\hline & & Orientin [40] \\
\hline & & Asparanin A [42] \\
\hline & & Gracillin [44] \\
\hline & \multirow{6}{*}{ Apoptosis } & Bulbine frutescens [37] \\
\hline & & Orientin $[40]$ \\
\hline & & Licochalcone A [41] \\
\hline & & Asparanin A [42] \\
\hline & & Parameritannin A-2 [43] \\
\hline & & Cernumidine [45] \\
\hline & \multirow{4}{*}{ Mitochondrial membrane potential imbalance } & Bullfrog oil [38] \\
\hline & & Rhein [39] \\
\hline & & Asparanin A [42] \\
\hline & & Cernumidine [45] \\
\hline \multirow{21}{*}{ Neurodegenerative diseases } & \multirow{4}{*}{ Energy metabolism obstruction } & Solanum melongena extract [46] \\
\hline & & Ganoderma lucidum [47] \\
\hline & & $\alpha$-Arbutin [51] \\
\hline & & Auraptene $[54]$ \\
\hline & \multirow{6}{*}{ Oxidative stress } & Solanum melongena extract [46] \\
\hline & & Linalool $[48]$ \\
\hline & & $\alpha$-Arbutin [51] \\
\hline & & Naringenin [52] \\
\hline & & Apigenin [53] \\
\hline & & Auraptene [54] \\
\hline & \multirow{7}{*}{ Apoptosis } & Ganoderma lucidum [47] \\
\hline & & Cinnamic acid derivatives [49] \\
\hline & & Proanthocyanidins [50] \\
\hline & & Naringenin [52] \\
\hline & & Apigenin [53] \\
\hline & & Naringenin [55] \\
\hline & & Ulmoside A [56] \\
\hline & \multirow{3}{*}{ Mitochondrial membrane potential imbalance } & Ganoderma lucidum [47] \\
\hline & & Linalool $[48]$ \\
\hline & & $\alpha$-Arbutin [51] \\
\hline & Mitochondrial fusion, division, and autophagy & Celastrol [57] \\
\hline \multirow{12}{*}{ Liver diseases } & \multirow{9}{*}{ Energy metabolism obstruction } & Rooibos tea $[58]$ \\
\hline & & Cimicifuga racemosa extract [59] \\
\hline & & Polygonatum kingianum [61] \\
\hline & & Betaine $[63]$ \\
\hline & & Nicotinamide riboside [64] \\
\hline & & Puerarin $[65]$ \\
\hline & & Punica granatum L. [62] \\
\hline & & Silybin [67] \\
\hline & & Salvianolic acid B [68] \\
\hline & \multirow{3}{*}{ Oxidative stress } & Cimicifuga racemosa extract [59] \\
\hline & & Sipjeondaebo-tang $[60]$ \\
\hline & & Polygonatum kingianum [61] \\
\hline
\end{tabular}


TABLE 8: Continued.

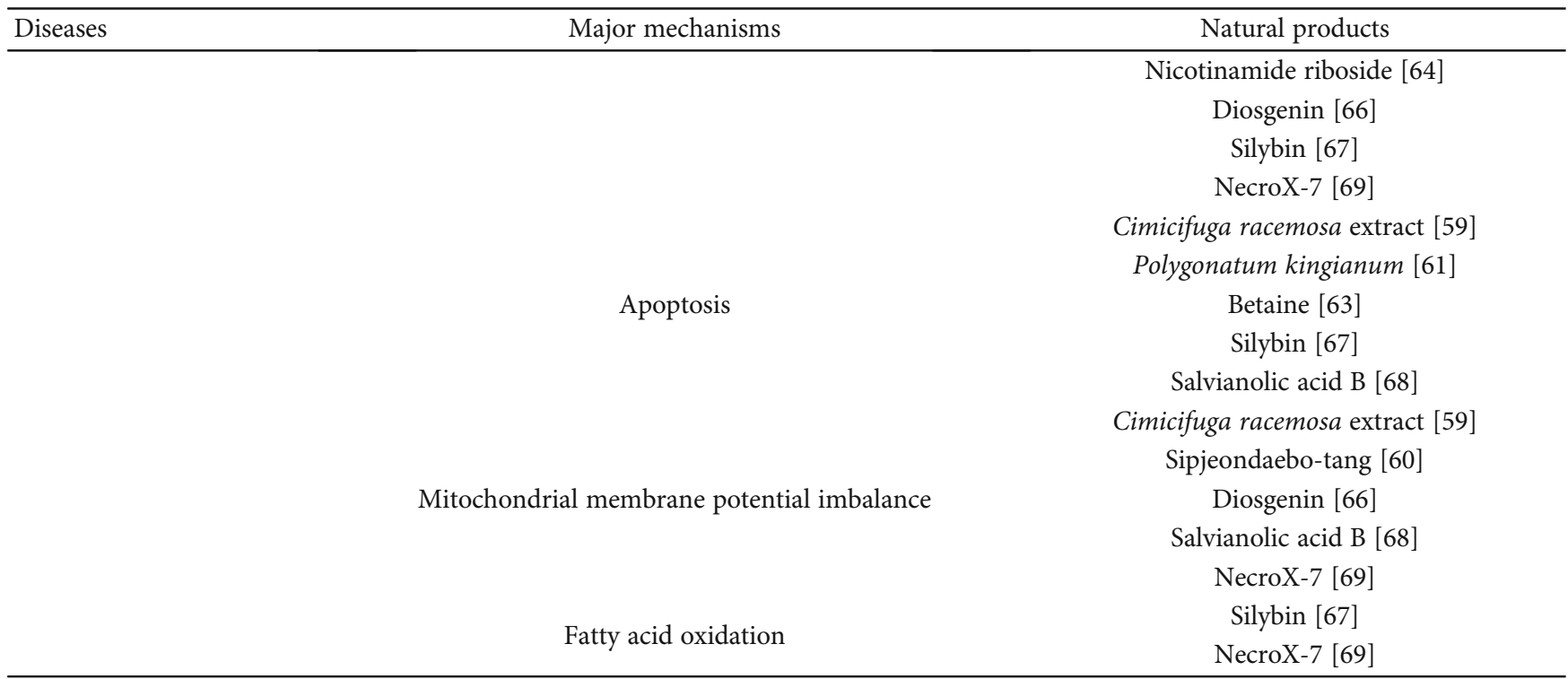

Polysaccharides from Portulaca oleracea L. [70] Korean red ginseng [71]

Energy metabolism obstruction

Berberine [72]

Quercetin [73]

Theaflavins [74]

Puerarin [76]

Mitochondrial membrane potential imbalance

Apoptosis

Mitochondrial fusion, division, and autophagy

Energy metabolism obstruction

Oxidative stress

Diabetes complications

Apoptosis

Mitochondrial membrane potential imbalance

Mitochondrial fusion, division, and autophagy
Polysaccharides from Portulaca oleracea L. [70] Silibinin [75] Silibinin [75]

Korean red ginseng [71]

Quercetin [73]

Theaflavins [74]

Silibinin [75]

Puerarin [76]

QiDiTangShen granules [77]

Shengmai San [78]

Water extracts of Liuwei Dihuang [79]

Salidroside [82]

Hydroxytyrosol [85]

Water extracts of Liuwei Dihuang [79]

Anthocyanins [80]

Ginsenoside Rb1 [84]

Hydroxytyrosol [85]

Anthocyanins [80]

Orientin [81]

Astragalus polysaccharides [83]

Ginsenoside Rb1 [84]

Orientin [81]

Water extracts of Liuwei Dihuang [79]

Orientin [81]

QiDiTangShen granules [77] Salidroside [82] 
TABLe 8: Continued.

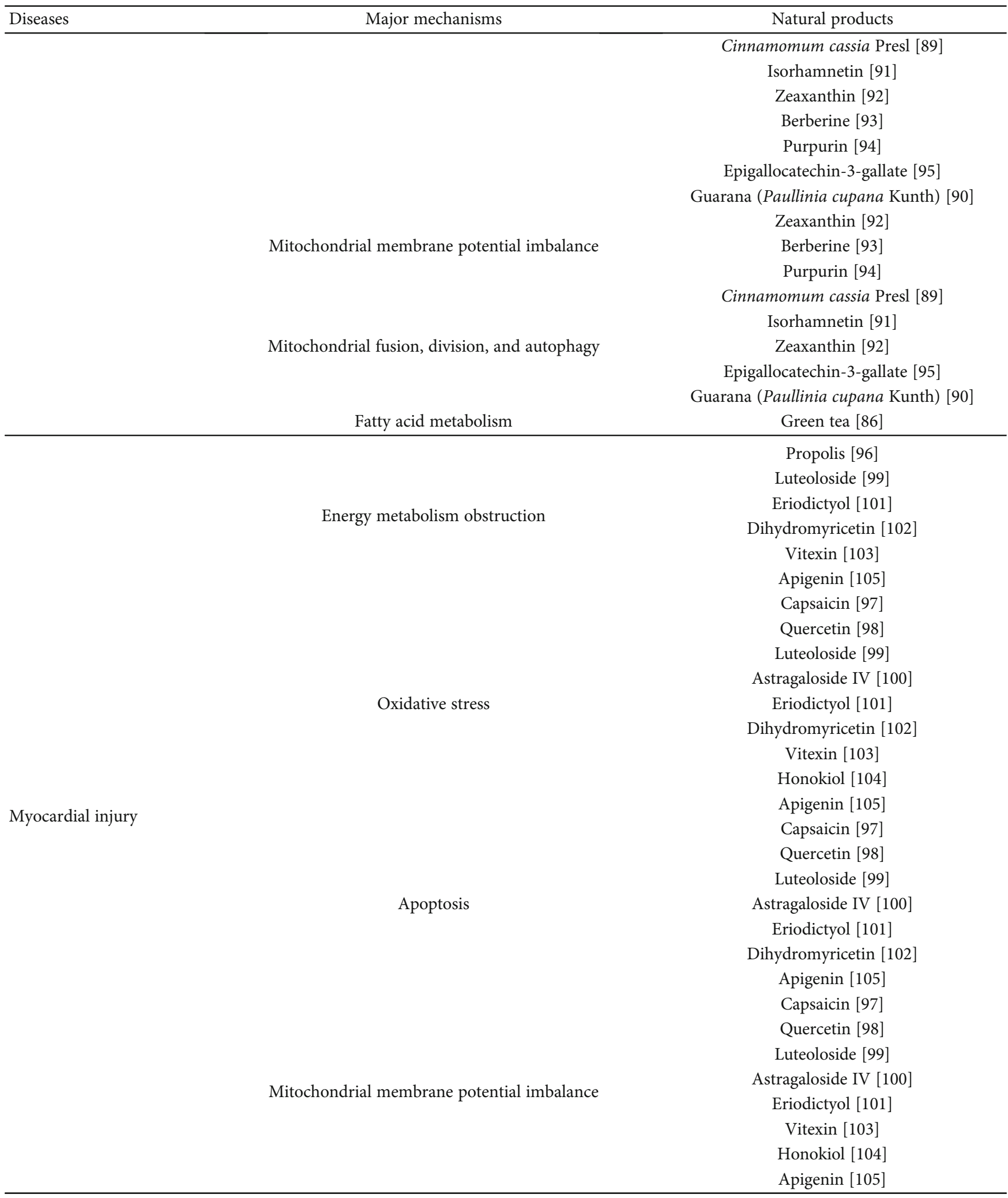




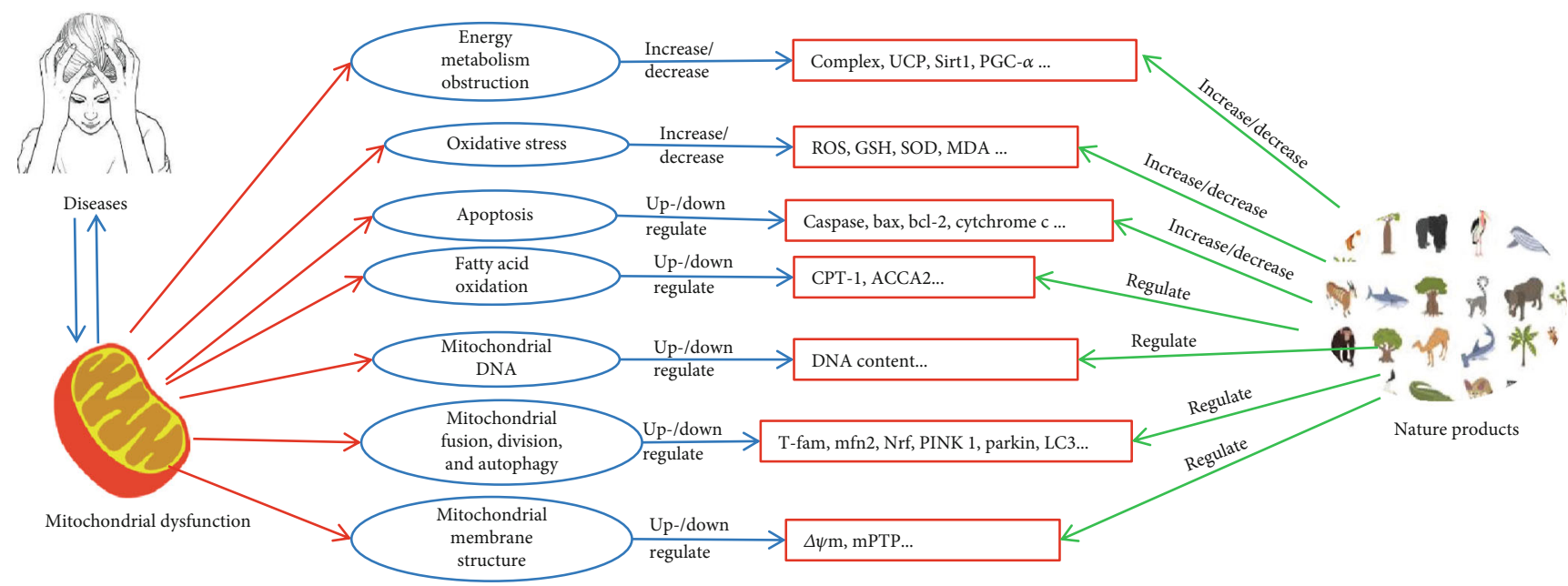

Figure 1: Remedying the mitochondria to cure human diseases by natural products.

\section{Abbreviations}

AFLD: Alcoholic fatty liver disease

ASH: Alcoholic steatohepatitis

DM: Diabetes mellitus

H/R: Hypoxia/reoxygenation

I/R: Ischemia/reperfusion

IR: Insulin resistance

LPS: Lipopolysaccharides

$\triangle \Psi \mathrm{m}: \quad$ Mitochondrial membrane potential

MPTP: 1-Methyl-4-phenyl-1,2,3,6-tetrahydropyridine

NAFLD: Nonalcoholic fatty liver disease

NASH: Nonalcoholic steatohepatitis

T1DM: Type 1 diabetes mellitus

T2DM: Type 2 diabetes mellitus.

\section{Data Availability}

My article is a summary, so there is no data to provide.

\section{Consent}

Publication of this manuscript has been approved by all co-authors.

\section{Conflicts of Interest}

The authors declare that there is no duality of interest associated with this manuscript.

\section{Authors' Contributions}

Jian-Kang $\mathrm{Mu}$ and Yan-Qin Li have contributed equally to this work.

\section{Acknowledgments}

This study was supported by grants from the National Natural Science Foundation of China (Grants 81660596, 81460623, and 81760733) and the Application and Basis
Research Project of Yunnan China (Grants 2019FF002-061 and 2017FF117-013).

\section{References}

[1] F. Wang, M.-A. Ogasawara, and P. Huang, "Small mitochondria-targeting molecules as anti-cancer agents," Molecular Aspects of Medicine, vol. 31, no. 1, pp. 75-92, 2010.

[2] J. R. Friedman and J. Nunnari, "Mitochondrial form and function," Nature, vol. 505, no. 7483, pp. 335-343, 2014.

[3] V. Eisner, M. Picard, and G. Hajnóczky, "Mitochondrial dynamics in adaptive and maladaptive cellular stress responses," Nature Cell Biology, vol. 20, no. 7, pp. 755-765, 2018.

[4] J. B. Spinelli and M. C. Haigis, "The multifaceted contributions of mitochondria to cellular metabolism," Nature Cell Biology, vol. 20, no. 7, pp. 745-754, 2018.

[5] Y. Wang, X. Fan, H. Qu, X. Gao, and Y. Cheng, "Strategies and techniques for multi-component drug design from medicinal herbs and traditional chinese medicine," Current Topics in Medicinal Chemistry, vol. 12, no. 12, pp. 13561362, 2012.

[6] B. Wang, J. Deng, Y. Gao, L. Zhu, R. He, and Y. Xu, "The screening toolbox of bioactive substances from natural products: A review," Fitoterapia, vol. 82, no. 8, pp. 1141-1151, 2011.

[7] S. Fulda, "Tumor resistance to apoptosis," International Journal of Cancer, vol. 124, no. 3, pp. 511-515, 2009.

[8] P. Golstein and G. Kroemer, "Cell death by necrosis: towards a molecular definition," Trends in Biochemical Sciences, vol. 32, no. 1, pp. 37-43, 2007.

[9] A.-V. Kudryavtseva, G.-S. Krasnov, A.-A. Dmitriev et al., "Mitochondrial dysfunction and oxidative stress in aging and cancer," Oncotarget, vol. 7, no. 29, pp. 44879-44905, 2016.

[10] J.-A. Petros, A.-K. Baumann, E. Ruiz-Pesini et al., "mtDNA mutations increase tumorigenicity in prostate cancer," Proceedings of the National Academy of Sciences of the United States of America, vol. 102, no. 3, pp. 719-724, 2005.

[11] G. Lou, K. Palikaras, S. Lautrup, M. Scheibye-Knudsen, N. Tavernarakis, and E. F. Fang, "Mitophagy and neuroprotection," Trends in Molecular Medicine, vol. 26, no. 1, pp. 8-20, 2020. 
[12] K. Panchal and A.-K. Tiwari, "Mitochondrial dynamics, a key executioner in neurodegenerative diseases," Mitochondrion, vol. 47, pp. 151-173, 2019.

[13] Y. Luo, A. Hoffer, B. Hoffer, and X. Qi, "Mitochondria: a therapeutic target for Parkinson's disease?," International Journal of Molecular Sciences, vol. 16, no. 9, pp. 20704-20730, 2015.

[14] X. Zhang, X. Wu, Q. Hu et al., "Mitochondrial DNA in liver inflammation and oxidative stress," Life Sciences, vol. 236, article 116464, 2019.

[15] E. Crosas-Molist and I. Fabregat, "Role of NADPH oxidases in the redox biology of liver fibrosis," Redox Biology, vol. 6, pp. 106-111, 2015.

[16] T.-A. Ajith, "Role of mitochondria and mitochondriatargeted agents in non-alcoholic fatty liver disease," Clinical and Experimental Pharmacology and Physiology, vol. 45, no. 5, pp. 413-421, 2018.

[17] A.-M. Schmidt, "Diabetes mellitus and cardiovascular disease," Arteriosclerosis, Thrombosis, and Vascular Biology, vol. 39, no. 4, pp. 558-568, 2019.

[18] S. Fujimaki and T. Kuwabara, "Diabetes-induced dysfunction of mitochondria and stem cells in skeletal muscle and the nervous system," International Journal of Molecular Sciences, vol. 18, no. 10, p. 2147, 2017.

[19] D. M. D'Souza, D. Al-Sajee, and T. J. Hawke, "Diabetic myopathy: impact of diabetes mellitus on skeletal muscle progenitor cells," Frontiers in Physiology, vol. 4, p. 379, 2013.

[20] F. Zatterale, M. Longo, J. Naderi et al., "Chronic adipose tissue inflammation linking obesity to insulin resistance and type 2 diabetes," Frontiers in Physiology, vol. 10, p. 1607, 2020.

[21] Y. Lin and Z. Sun, "Current views on type 2 diabetes," The Journal of Endocrinology, vol. 204, no. 1, pp. 1-11, 2010.

[22] L. Vilarinho, F.-M. Santorelli, I. Coelho et al., "The mitochondrial DNA A3243G mutation in Portugal: clinical and molecular studies in 5 families," Journal of the Neurological Sciences, vol. 163, no. 2, pp. 168-174, 1999.

[23] E. Heyman, F. Daussin, V. Wieczorek et al., "Muscle oxygen supply and use in type 1 diabetes, from ambient air to the mitochondrial respiratory chain: is there a limiting step?," Diabetes Care, vol. 43, no. 1, pp. 209-218, 2020.

[24] T. Jelenik, U. Flögel, E. Álvarez-Hernández et al., "Insulin resistance and vulnerability to cardiac ischemia," Diabetes, vol. 67, no. 12, pp. 2695-2702, 2018.

[25] N.-B. Flemming, L.-A. Gallo, M.-S. Ward, and J.-M. Forbes, "Tapping into mitochondria to find novel targets for diabetes complications," Current Drug Targets, vol. 17, no. 12, pp. 1341-1349, 2016.

[26] R.-A. Kowluru, "Mitochondrial stability in diabetic retinopathy: lessons learned from epigenetics," Diabetes, vol. 68, no. 2, pp. 241-247, 2019.

[27] R. Blake and I.-A. Trounce, "Mitochondrial dysfunction and complications associated with diabetes," Biochimica et Biophysica Acta (BBA) - General Subjects, vol. 1840, no. 4, pp. 1404-1412, 2014.

[28] P.-Z. Wei and C.-C. Szeto, "Mitochondrial dysfunction in diabetic kidney disease," Clinica Chimica Acta, vol. 496, pp. 108-116, 2019.

[29] E. Björnson, M. Adiels, M.-R. Taskinen, and J. Borén, "Kinetics of plasma triglycerides in abdominal obesity," Current Opinion in Lipidology, vol. 28, no. 1, pp. 1-18, 2016.
[30] M. Blüher, "Adipose tissue dysfunction contributes to obesity related metabolic diseases," Best Practice \& Research. Clinical Endocrinology \& Metabolism, vol. 27, no. 2, pp. 163-177, 2013.

[31] A.-H. de Mello, A.-B. Costa, J.-D.-G. Engel, and G.-T. Rezin, "Mitochondrial dysfunction in obesity," Life Sciences, vol. 192, pp. 26-32, 2018.

[32] C.-S. Lai, J.-C. Wu, C.-T. Ho, and M.-H. Pan, "Chemoprevention of obesity by dietary natural compounds targeting mitochondrial regulation," Molecular Nutrition \& Food Research, vol. 61, no. 6, 2017.

[33] S. Hernandez-Resendiz, K. Chinda, S.-B. Ong, H. CabreraFuentes, C. Zazueta, and D. J. Hausenloy, "The role of redox dysregulation in the inflammatory response to acute myocardial ischaemia-reperfusion injury - adding fuel to the fire," Current Medicinal Chemistry, vol. 25, no. 11, pp. 12751293, 2018.

[34] M. Neri, I. Riezzo, N. Pascale, C. Pomara, and E. Turillazzi, "Ischemia/reperfusion injury following acute myocardial infarction: a critical issue for clinicians and forensic pathologists," Mediators of Inflammation, vol. 2017, Article ID 7018393, 14 pages, 2017.

[35] J.-L. Pohjoismäki and S. Goffart, "The role of mitochondria in cardiac development and protection," Free Radical Biology \& Medicine, vol. 106, pp. 345-354, 2017.

[36] M. Jašová, I. Kancirová, I. Waczulíková, and M. Ferko, “Mitochondria as a target of cardioprotection in models of preconditioning," Journal of Bioenergetics and Biomembranes, vol. 49, no. 5, pp. 357-368, 2017.

[37] P. P. Kushwaha, P. S. Vardhan, P. Kapewangolo et al., "Bulbine frutescens phytochemical inhibits notch signaling pathway and induces apoptosis in triple negative and luminal breast cancer cells," Life Sciences, vol. 234, article 116783, 2019.

[38] L. Amaral-Machado, W. N. Oliveira, É. N. Alencar et al., "Bullfrog oil (Rana catesbeiana Shaw) induces apoptosis, in A2058 human melanoma cells by mitochondrial dysfunction triggered by oxidative stress," Biomedicine \& Pharmacotherapy, vol. 117, article 109103, 2019.

[39] L. Wu, K. Cao, Z. Ni et al., "Rhein reverses doxorubicin resistance in SMMC-7721 liver cancer cells by inhibiting energy metabolism and inducing mitochondrial permeability transition pore opening," BioFactors, vol. 45, no. 1, pp. 85-96, 2019.

[40] K. Thangaraj, B. Balasubramanian, S. Park, K. Natesan, W. Liu, and V. Manju, "Orientin induces G0/G1 cell cycle arrest and mitochondria mediated intrinsic apoptosis in human colorectal carcinoma HT29 cells," Biomolecules, vol. 9, no. 9, p. 418, 2019.

[41] S. H. Hong, H.-J. Cha, H. Hwang-Bo et al., "Anti-proliferative and pro-apoptotic effects of licochalcone a through ROSmediated cell cycle arrest and apoptosis in human bladder cancer cells," International Journal of Molecular Sciences, vol. 20, no. 15, p. 3820, 2019.

[42] F. Zhang, Y.-Y. Zhang, Y.-S. Sun et al., "Asparanin a from asparagus officinalis L. induces G0/G1 cell cycle arrest and apoptosis in human endometrial carcinoma ishikawa cells via mitochondrial and PI3K/AKT signaling pathways," Journal of Agricultural and Food Chemistry, vol. 68, no. 1, pp. 213-224, 2019.

[43] L. Liang, A. Amin, W.-Y. Cheung et al., "Parameritannin A-2 from Urceola huaitingii enhances doxorubicin-induced mitochondria-dependent apoptosis by inhibiting the 
PI3K/Akt, ERK1/2 and p38 pathways in gastric cancer cells," Chemico-Biological Interactions, vol. 316, article 108924, 2020.

[44] H.-Y. Min, H.-J. Jang, K. H. Park et al., "The natural compound gracillin exerts potent antitumor activity by targeting mitochondrial complex II," Cell Death \& Disease, vol. 10, no. 11, p. 810, 2019.

[45] M. A. Miranda, A. Mondal, M. Sachdeva et al., "Chemosensitizing effect of cernumidine extracted from solanum cernuum on bladder cancer cells in vitro," Chemistry \& Biodiversity, vol. 16, no. 10, article e1900334, 2019.

[46] Y. Youn, S.-. H. Jeon, H.-. Y. Jin, D. N. Che, S.-. I. Jang, and Y.-. S. Kim, "Chlorogenic acid-rich Solanum melongena extract has protective potential against rotenone-induced neurotoxicity in PC-12 cells," Journal of Food Biochemistry, vol. 43, no. 11, article e12999, 2019.

[47] Z.-1. Ren, C.-d. Wang, T. Wang et al., "Ganoderma lucidum extract ameliorates MPTP-induced parkinsonism and protects dopaminergic neurons from oxidative stress via regulating mitochondrial function, autophagy, and apoptosis," Acta Pharmacologica Sinica, vol. 40, no. 4, pp. 441-450, 2019.

[48] A. M. Sabogal-Guáqueta, F. Hobbie, A. Keerthi et al., "Linalool attenuates oxidative stress and mitochondrial dysfunction mediated by glutamate and NMDA toxicity," Biomedicine \& Pharmacotherapy, vol. 118, article 109295, 2019.

[49] W.-X. Zhang, H. Wang, H.-R. Cui et al., "Design, synthesis and biological evaluation of cinnamic acid derivatives with synergetic neuroprotection and angiogenesis effect," European Journal of Medicinal Chemistry, vol. 183, article 111695, 2019.

[50] J. Zhang, X. Zhang, C. Wen, Y. Duan, and H. Zhang, "Lotus seedpod proanthocyanidins protect against neurotoxicity after methyl- mercuric chloride injury," Ecotoxicology and Environmental Safety, vol. 183, article 109560, 2019.

[51] Y. Ding, D. Kong, T. Zhou et al., “ $\alpha$-Arbutin protects against Parkinson's disease-associated mitochondrial dysfunction in vitro and in vivo," Neuromolecular Medicine, vol. 22, no. 1, pp. 56-67, 2020.

[52] A.-M. Krishna Chandran, H. Christina, S. Das, K.D. Mumbrekar, and B.-S. Satish Rao, "Neuroprotective role of naringenin against methylmercury induced cognitive impairment and mitochondrial damage in a mouse model," Environmental Toxicology and Pharmacology, vol. 71, article 103224, 2019.

[53] F. Zhao, Y. Dang, R. Zhang et al., "Apigenin attenuates acrylonitrile-induced neuro-inflammation in rats: involved of inactivation of the TLR4/NF- $\kappa \mathrm{B}$ signaling pathway," International Immunopharmacology, vol. 75, article 105697, 2019.

[54] Y. Jang, H. Choo, M. J. Lee et al., "Auraptene mitigates Parkinson's disease-like behavior by protecting inhibition of mitochondrial respiration and scavenging reactive oxygen species," International Journal of Molecular Sciences, vol. 20, no. 14, p. 3409, 2019.

[55] Y. Jin and H. Wang, "Naringenin inhibit the hydrogen peroxide-induced SH-SY5Y cells injury through Nrf 2/HO1 pathway," Neurotoxicity Research, vol. 36, no. 4, pp. 796805, 2019.

[56] P. Gupta, A. Singh, S. Tiwari, A. Mishra, R. Maurya, and S. Singh, "Ulmosides A: flavonoid 6-C-glycosides from ulmus wallichiana attenuates lipopolysacchride induced oxidative stress, apoptosis and neuronal death," NeuroToxicology, vol. 73, pp. 100-111, 2019.
[57] M.-W. Lin, C. C. Lin, Y.-H. Chen, H.-B. Yang, and S.Y. Hung, "Celastrol inhibits dopaminergic neuronal death of Parkinson's disease through activating mitophagy," Antioxidants, vol. 9, no. 1, p. 37, 2020.

[58] O. Uličná, O. Vančová, J. Kucharská, P. Janega, and I. Waczulíková, "Rooibos tea (Aspalathus linearis) ameliorates the $\mathrm{CCl} 4$-induced injury to mitochondrial respiratory function and energy production in rat liver," General Physiology and Biophysics, vol. 38, no. 1, pp. 15-25, 2019.

[59] M. Rabenau, M. Unger, J. Drewe, and C. Culmsee, "Metabolic switch induced by Cimicifuga racemosa extract prevents mitochondrial damage and oxidative cell death," Phytomedicine, vol. 52, pp. 107-116, 2019.

[60] S. M. Park, S. W. Kim, E. H. Jung et al., "Sipjeondaebo-tang qlleviates oxidative stress-mediated liver injury through activation of the CaMKK2-AMPK signaling pathway," EvidenceBased Complementary and Alternative Medicine, vol. 2018, Article ID 8609285, 13 pages, 2018.

[61] X.-X. Yang, X. Wang, T.-T. Shi et al., "Mitochondrial dysfunction in high-fat diet-induced nonalcoholic fatty liver disease: The alleviating effect and its mechanism of Polygonatum kingianum," Biomedicine \& Pharmacotherapy, vol. 117, article 109083, 2019.

[62] X. Zou, C. Yan, Y. Shi et al., "Mitochondrial dysfunction in obesity-associated nonalcoholic fatty liver disease: the protective effects of pomegranate with its active component punicalagin," Antioxidants \& Redox Signaling, vol. 21, no. 11, pp. 1557-1570, 2014.

[63] M. Jung Kim, "Betaine enhances the cellular survival via mitochondrial fusion and fission factors, MFN2 and DRP1," Animal Cells and Systems, vol. 22, no. 5, pp. 289-298, 2018.

[64] S. Wang, T. Wan, M. Ye et al., "Nicotinamide riboside attenuates alcohol induced liver injuries via activation of SirT1/PGC- $1 \alpha /$ mitochondrial biosynthesis pathway," Redox Biology, vol. 17, pp. 89-98, 2018.

[65] S. Wang, F.-J. Yang, L.-C. Shang, Y. H. Zhang, Y. Zhou, and X. L. Shi, "Puerarin protects against high-fat high-sucrose diet-induced non-alcoholic fatty liver disease by modulating PARP-1/PI3K/AKT signaling pathway and facilitating mitochondrial homeostasis," Phytotherapy Research, vol. 33, no. 9, pp. 2347-2359, 2019.

[66] K. Fang, F. Wu, G. Chen et al., "Diosgenin ameliorates palmitic acid-induced lipid accumulation via AMPK/ACC/CPT$1 \mathrm{~A}$ and SREBP-1c/FAS signaling pathways in LO2 cells," $B M C$ Complementary and Alternative Medicine, vol. 19, no. 1, p. 255, 2019.

[67] G. Vecchione, E. Grasselli, F. Cioffi et al., "The nutraceutic silybin counteracts excess lipid accumulation and ongoing oxidative stress in an in vitro model of non-alcoholic fatty liver disease progression," Frontiers in Nutrition, vol. 4, p. $42,2017$.

[68] Y.-C. Wang, W.-Z. Kong, Q.-M. Jin, J. Chen, and L. Dong, "Effects of salvianolic acid B on liver mitochondria of rats with nonalcoholic steatohepatitis," World Journal of Gastroenterology, vol. 21, no. 35, pp. s10104-s10112, 2015.

[69] H.-K. Chung, Y.-K. Kim, J.-H. Park et al., "The indole derivative necrox-7 improves nonalcoholic steatohepatitis in ob/ob mice through suppression of mitochondrial ROS/RNS and inflammation," Liver International, vol. 35, no. 4, pp. 1341-1353, 2015.

[70] Q. Hu, Q. Niu, H. Song et al., "Polysaccharides from Portulaca oleracea L. regulated insulin secretion in INS-1 cells 
through voltage-gated $\mathrm{Na}{ }^{+}$channel," Biomedicine \& Pharmacotherapy, vol. 109, pp. 876-885, 2019.

[71] J.-K. Park, J.-Y. Shim, A.-R. Cho, M.-R. Cho, and Y.-J. Lee, "Korean red ginseng protects against mitochondrial damage and intracellular inflammation in an animal model of type 2 diabetes mellitus," Journal of Medicinal Food, vol. 21, no. 6, pp. 544-550, 2018.

[72] M. Javadipour, M. Rezaei, E. Keshtzar, and M.-J. Khodayar, "Metformin in contrast to berberine reversed arsenicinduced oxidative stress in mitochondria from rat pancreas probably via Sirt3-dependent pathway," Journal of Biochemical and Molecular Toxicology, vol. 33, no. 9, 2019.

[73] M.-J. Houghton, A. Kerimi, S. Tumova, J.-P. Boyle, and G. Williamson, "Quercetin preserves redox status and stimulates mitochondrial function in metabolically-stressed HepG2 cells," Free Radical Biology \& Medicine, vol. 129, pp. 296-309, 2018.

[74] T. Tong, N. Ren, P. Soomi et al., "Theaflavins improve insulin sensitivity through regulating mitochondrial biosynthesis in palmitic acid-induced HepG2 cells," Molecules, vol. 23, no. 12, p. 3382, 2018.

[75] Y. Sun, J. Yang, W. Liu et al., "Attenuating effect of silibinin on palmitic acid-induced apoptosis and mitochondrial dysfunction in pancreatic $\beta$-cells is mediated by estrogen receptor alpha," Molecular and Cellular Biochemistry, vol. 460, no. 1-2, pp. 81-92, 2019.

[76] X.-F. Chen, L. Wang, Y.-Z. Wu et al., "Effect of puerarin in promoting fatty acid oxidation by increasing mitochondrial oxidative capacity and biogenesis in skeletal muscle in diabetic rats," Nutrition \& Diabetes, vol. 8, no. 1, 2018.

[77] X. Wang, L. Zhao, A.-K. Ajay et al., "QiDiTangShen granules activate renal nutrient-sensing associated autophagy in $\mathrm{db} / \mathrm{db}$ mice," Frontiers in Physiology, vol. 10, p. 1224, 2019.

[78] J. Tian, W. Tang, M. Xu et al., "Shengmai san alleviates diabetic cardiomyopathy through improvement of mitochondrial lipid metabolic disorder," Cellular Physiology and Biochemistry, vol. 50, no. 5, pp. 1726-1739, 2018.

[79] Y.-T. Tseng, W.-H. Chang, C.-C. Lin, F. R. Chang, P. C. Wu, and Y. C. Lo, "Protective effects of Liuwei Dihuang water extracts on diabetic muscle atrophy," Phytomedicine, vol. 53, pp. 96-106, 2019.

[80] J. Wei, H. Wu, H. Zhang et al., "Anthocyanins inhibit high glucose-induced renal tubular cell apoptosis caused by oxidative stress in $\mathrm{db} / \mathrm{db}$ mice," International Journal of Molecular Medicine, vol. 41, no. 3, pp. 1608-1618, 2018.

[81] Z.-L. Kong, K. Che, J.-X. Hu et al., "Orientin protects podocytes from high glucose induced apoptosis through mitophagy," Chemistry \& Biodiversity, vol. 17, no. 3, article e1900647, 2020.

[82] H. Xue, P. Li, Y. Luo et al., "Salidroside stimulates the Sirt1/PGC- $1 \alpha$ axis and ameliorates diabetic nephropathy in mice," Phytomedicine, vol. 54, pp. 240-247, 2019.

[83] S. Sun, S. Yang, M. Dai et al., "The effect of Astragalus polysaccharides on attenuation of diabetic cardiomyopathy through inhibiting the extrinsic and intrinsic apoptotic pathways in high glucose -stimulated H9C2 cells," BMC Complementary and Alternative Medicine, vol. 17, no. 1, p. 310, 2017.

[84] F. Nan, G. Sun, W. Xie et al., "Ginsenoside Rb1 mitigates oxidative stress and apoptosis induced by methylglyoxal in SH-SY5Y cells via the PI3K/Akt pathway," Molecular and Cellular Probes, vol. 48, article 101469, 2019.
[85] A. Zheng, H. Li, J. Xu et al., "Hydroxytyrosol improves mitochondrial function and reduces oxidative stress in the brain of $\mathrm{db} / \mathrm{db}$ mice: role of AMP-activated protein kinase activation," The British Journal of Nutrition, vol. 113, no. 11, pp. 1667-1676, 2015.

[86] L.-S. Lee, J.-H. Choi, M.-J. Sung et al., "Green tea changes serum and liver metabolomic profiles in mice with high-fat diet-induced obesity," Molecular Nutrition \& Food Research, vol. 59, no. 4, pp. 784-794, 2015.

[87] S. Seo, S.-M. Jo, J. Kim, M. Lee, Y. Lee, and I. Kang, "Peanut sprout extracts attenuate triglyceride accumulation by promoting mitochondrial fatty acid oxidation in adipocytes," International Journal of Molecular Sciences, vol. 20, no. 5, p. 1216, 2019.

[88] T. Yoneshiro, R. Kaede, K. Nagaya et al., "Melinjo (Gnetum gnemon $\mathrm{L}$ ) seed extract induces uncoupling protein 1 expression in brown fat and protects mice against diet-induced obesity, inflammation, and insulin resistance," Nutrition Research, vol. 58, pp. 17-25, 2018.

[89] M.-Y. Song, S.-Y. Kang, A. Kang, J. H. Hwang, Y. K. Park, and H. W. Jung, "Cinnamomum cassia Prevents high-fat dietinduced obesity in mice through the increase of muscle energy," The American Journal of Chinese Medicine, vol. 45, no. 5, pp. 1017-1031, 2017.

[90] N.-D.-S. Lima, L. Teixeira, A. Gambero, and M.-L. Ribeiro, "Guarana (Paullinia cupana) stimulates mitochondrial biogenesis in mice fed high-fat diet," Nutrients, vol. 10, no. 2, 2018.

[91] M.-S. Lee and Y. Kim, "Effects of isorhamnetin on adipocyte mitochondrial biogenesis and AMPK activation," Molecules, vol. 23, no. 8, p. 1853, 2018.

[92] M. Liu, M. Zheng, D. Cai et al., "Zeaxanthin promotes mitochondrial biogenesis and adipocyte browningviaAMPK $\alpha 1$ activation," Food \& Function, vol. 10, no. 4, pp. 2221-2233, 2019.

[93] Y. Sun, C. Jin, X. Zhang, W. Jia, J. le, and J. Ye, "Restoration of GLP-1 secretion by berberine is associated with protection of colon enterocytes from mitochondrial overheating in dietinduced obese mice," Nutrition \& Diabetes, vol. 8, no. 1, p. 53, 2018.

[94] W. Nam, S.-H. Nam, S.-P. Kim, C. Levin, and M. Friedman, "Anti-adipogenic and anti-obesity activities of purpurin in 3T3-L1 preadipocyte cells and in mice fed a high-fat diet," BMC Complementary and Alternative Medicine, vol. 19, no. 1, p. 364, 2019.

[95] M.-S. Lee, Y. Shin, S. Jung, and Y. Kim, "Effects of epigallocatechin-3-gallate on thermogenesis and mitochondrial biogenesis in brown adipose tissues of diet-induced obese mice," Food \& Nutrition Research, vol. 61, no. 1, article 1325307, 2017.

[96] A. Braik, M. Lahouel, R. Merabe, M.-R. Djebar, and D. Morin, "Myocardial protection by propolis during prolonged hypothermic preservation," Cryobiology, vol. 88, pp. 29-37, 2019.

[97] J. Huang, Z. Liu, P. Xu et al., "Capsaicin prevents mitochondrial damage, protects cardiomyocytes subjected to anoxia/reoxygenation injury mediated by $14-3-3 \eta / \mathrm{Bcl}-2$," European Journal of Pharmacology, vol. 819, pp. 43-50, 2018.

[98] X. Chen, X. Peng, Y. Luo et al., "Quercetin protects cardiomyocytes against doxorubicin-induced toxicity by suppressing oxidative stress and improving mitochondrial function via 14-3-3 $\gamma$," Toxicology Mechanisms and Methods, vol. 29, no. 5, pp. 344-354, 2019. 
[99] Z. Liu, L. Yang, J. Huang et al., "Luteoloside attenuates anoxia/reoxygenation-induced cardiomyocytes injury via mitochondrial pathway mediated by $14-3-3 \eta$ protein," Phytotherapy Research, vol. 32, no. 6, pp. 1126-1134, 2018.

[100] Y. Luo, Q. Wan, M. Xu et al., "Nutritional preconditioning induced by astragaloside IV on isolated hearts and cardiomyocytes against myocardial ischemia injury via improving Bcl-2-mediated mitochondrial function," Chemico-Biological Interactions, vol. 309, article 108723, 2019.

[101] Y. Xie, R. Ji, and M. Han, "Eriodictyol protects H9c2 cardiomyocytes against the injury induced by hypoxia/reoxygenation by improving the dysfunction of mitochondria," Experimental and Therapeutic Medicine, vol. 17, no. 1, pp. 551-557, 2019.

[102] L. Wei, X. Sun, X. Qi, Y. Zhang, Y. Li, and Y. Xu, "Dihydromyricetin ameliorates cardiac ischemia/reperfusion injury through Sirt3 activation," Bio Med Research International, vol. 2019, article 6803943, pp. 1-9, 2019.

[103] W. Xue, X. Wang, H. Tang et al., "Vitexin attenuates myocardial ischemia/reperfusion injury in rats by regulating mitochondrial dysfunction induced by mitochondrial dynamics imbalance," Biomedicine \& Pharmacotherapy, vol. 124, article 109849, 2020.

[104] Z. Tan, H. Liu, X. Song et al., "Honokiol post-treatment ameliorates myocardial ischemia/reperfusion injury by enhancing autophagic flux and reducing intracellular ROS production," Chemico-Biological Interactions, vol. 307, pp. 82-90, 2019.

[105] H. Huang, S. Lai, Y. Luo et al., "Nutritional preconditioning of apigenin alleviates myocardial ischemia/reperfusion injury via the mitochondrial pathway mediated by notch $1 /$ hes 1 ," Oxidative Medicine and Cellular Longevity, vol. 2019, Article ID 7973098, 15 pages, 2019. 